\title{
Abundance stratification and pulsation in the atmosphere of the roAp star $\gamma$ Equulei $^{\star}$
}

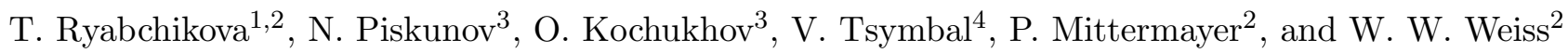 \\ 1 Institute of Astronomy, Russian Academy of Sciences, Pyatnitskaya 48, 109017 Moscow, Russia \\ e-mail: ryabchik@inasan.rssi.ru \\ 2 Institute for Astronomy, University of Vienna, Türkenschanzstrasse 17, 1180 Wien, Austria \\ e-mail: last_name@astro.univie.ac.at \\ 3 Uppsala Astronomical Observatory, Box 515, 751 20, Sweden \\ e-mail: piskunov@astro.uu.se, oleg@astro.uu.se \\ 4 Tavrian National University, Yaltinskaya 4, 330000 Simferopol, Crimea, Ukraine \\ e-mail: vad@ccssu.crimea.ua
}

Received 11 October 2001 / Accepted 10 January 2002

\begin{abstract}
We present the evidence for abundance stratification in the atmosphere of the rapidly oscillating Ap star $\gamma$ Equ. $\mathrm{Ca}, \mathrm{Cr}, \mathrm{Fe}, \mathrm{Ba}, \mathrm{Si}, \mathrm{Na}$ seem to be overabundant in deeper atmospheric layers, but normal to underabundant in the upper layers with a transition in the typical line forming region of $-1.5<\log \tau_{5000}<-0.5$. This stratification profile agrees well with diffusion theory for $\mathrm{Ca}$ and $\mathrm{Cr}$, developed for cool magnetic stars with a weak mass loss of $\approx 2.5 \times 10^{-15} M_{\odot} \mathrm{yr}^{-1}$. Pr and Nd from the rare earth elements have an opposite profile. Their abundance is more than 6 dex higher above $\log \tau_{5000} \approx-8.0$ than in the deeper atmospheric layers. We further discuss the implications of abundance stratification in the context of radial velocity amplitudes and phases observed by Kochukhov \& Ryabchikova (2001) for a variety of spectral lines and elements using high spectral and time resolved, high $S / N$ observations.
\end{abstract}

Key words. stars: chemically peculiar - stars: abundances - stars: oscillations - stars: individual: $\gamma$ Equ

\section{Introduction}

$\gamma$ Equ is one of the brightest pulsating chemically peculiar (roAp) stars with a period of about $12.3 \mathrm{~min}$ (Martinez et al. 1996) in brightness as well as in radial velocity $(R V)$. Libbrecht (1988) was the first to find $R V$ pulsational variations in $\gamma$ Equ with an amplitude $42 \pm 15 \mathrm{~m} \mathrm{~s}^{-1}$. Later Kanaan \& Hatzes (1998) performed line-by-line $R V$ analysis and obtained that $R V$ amplitudes varied from $\approx 25 \mathrm{~m} \mathrm{~s}^{-1}$ to $400-600 \mathrm{~m} \mathrm{~s}^{-1}$, and reached $1 \mathrm{~km} \mathrm{~s}^{-1}$ in one case! Malanushenko et al. (1998) and Savanov et al. (1999) analysed $R V$ amplitudes of individual lines in the 6112-6172 $\AA$ spectral region and found that strong Pr III and Nd III lines showed the highest $R V$ amplitudes of up to $800 \mathrm{~m} \mathrm{~s}^{-1}$, while $R V$ amplitudes from strong and weak lines of other elements did not exceed the error limit of $150 \mathrm{~m} \mathrm{~s}^{-1}$. This result was recently confirmed

Send offprint requests to: W. W. Weiss,

e-mail: weiss@astro.univie.ac.at

* Based on observations obtained at the European Southern Observatory (La Silla, Chile), and the South African Astronomical Observatory. by Kochukhov \& Ryabchikova (2001, hereafter PaperI) using spectra with much higher spectral resolution and $S / N$ ratio. An error in their $R V$ measurements for unblended lines, $15-20 \mathrm{~m} \mathrm{~s}^{-1}$, was comparable to Libbrecht's and Kanaan \& Hatzes' data. They obtained pulsation signatures for 29 features in the 6140-6166 $\AA$ spectral region and also found $R V$ phase shifts between Pr III and Nd III lines relative to lines of other elements. $R V$ amplitudes obtained for the lines of singly-ionized rare-earth elements (REE) were higher than for the lines of lighter elements, but 1.5-2 times lower than for doubly ionized REE elements.

For $\gamma$ Equ Cowley \& Bord (1998) found the Nd abundance obtained from lines of the second ions to be higher by 1.5 dex than from lines of the first ions. Ryabchikova et al. (2001) and Weiss et al. (2000) analysed Pr III and Nd III lines in spectra of roAp and non-pulsating Ap stars, including $\gamma$ Equ. They confirmed the Nd anomaly in $\gamma$ Equ and found the same effect for $\mathrm{Pr}$, what seemed to be a typical feature of all roAp stars investigated so far. This anomaly is marginally present in non-pulsating stars, if at all. Ryabchikova et al. (2000) argue that in the cooler roAp 
stars HD 122970 and 10 Aql the observed Pr-Nd anomaly may be due to a high concentration of these elements in the uppermost atmospheric layers.

In stellar atmospheres, stabilized by magnetic fields, radiation pressure and gravitational settling will gradually generate a superficial atmosphere with peculiar chemical composition (Michaud 1970). Babel (1992) calculated Ca, $\mathrm{Ti}, \mathrm{Cr}, \mathrm{Mn}$ diffusion in the atmosphere of a magnetic star in the presence of a weak stellar wind and showed that strong vertical abundance gradients can occur in the line forming region. His model explained the anomalous line profiles in the Ap star $53 \mathrm{Cam}$, in particular for $\mathrm{Ca}$, and the author later showed (Babel 1994) that Ca is stratified in the atmospheres of a large sample of Ap stars with $T_{\text {eff }}<9000 \mathrm{~K}$.

Hence, abundance stratification together with pulsation signatures of individual lines may help to investigate the 3-D pulsation velocity field in roAp stars. Baldry et al. (1999) studied with low-resolution spectra pulsation effects across the $\mathrm{H} \alpha$ profile of the roAp star $\alpha \mathrm{Cir}$ and claimed to have found evidence for a radial node. Later Baldry \& Bedding (2000) repeated the analysis for another roAp star, HR 3831 (HD 83368). For both stars bisector shifts at different relative intensities of the $\mathrm{H} \alpha$ profile (at different heights in the stellar atmosphere) were measured. The pulsation period of HR 3831 is about twice the period of $\alpha$ Cir. Therefore, a variation of $R V$ amplitudes due to standing waves, measured at different relative depths in the $\mathrm{H} \alpha$ profile, should be different for the two stars, if they have similar atmospheres (what is indicated from spectroscopy). However, for both stars the authors obtained a very similar distribution of $R V$ amplitudes and phases across the $\mathrm{H} \alpha$ profile and they considered this to be an argument against the existence of a radial node.

In this paper we study the abundance stratification in the atmosphere of the roAp star $\gamma$ Equ. Together with results from an already mentioned high-spectral and time resolved spectroscopy, it is possible to propose a vertical distribution of pulsation amplitudes in the atmosphere of $\gamma$ Equ.

\section{Observations and spectra reduction}

A high-resolution spectrum in the $6140-6166 \AA$ region was obtained by coadding 31 time-resolved spectra acquired with the CES spectrograph fiber linked to the ESO 3.6-meter telescope what resulted in a resolution $R$ of about $\lambda / \Delta \lambda=166000$ and $S / N=800$ (see Paper I). We also used a CAT + CES spectrum (4266-4285 $\AA$ spectral range, and $R=80000$ (see Ryabchikova et al. 1997, hereafter Paper II) and spectra obtained at the Dominion Astrophysical Observatory with a resolution of 50000 (Paper II). In addition, an echelle spectrum of $\gamma$ Equ was obtained at the South African Astronomical Observatory (SAAO) with the MUSICOS-type spectrograph GIRAFFE. It provided a spectrum in the $4700-7100 \AA$ region with a medium resolution of 35000 and $S / N=300$ which was reduced with IRAF. Special attention was payed to

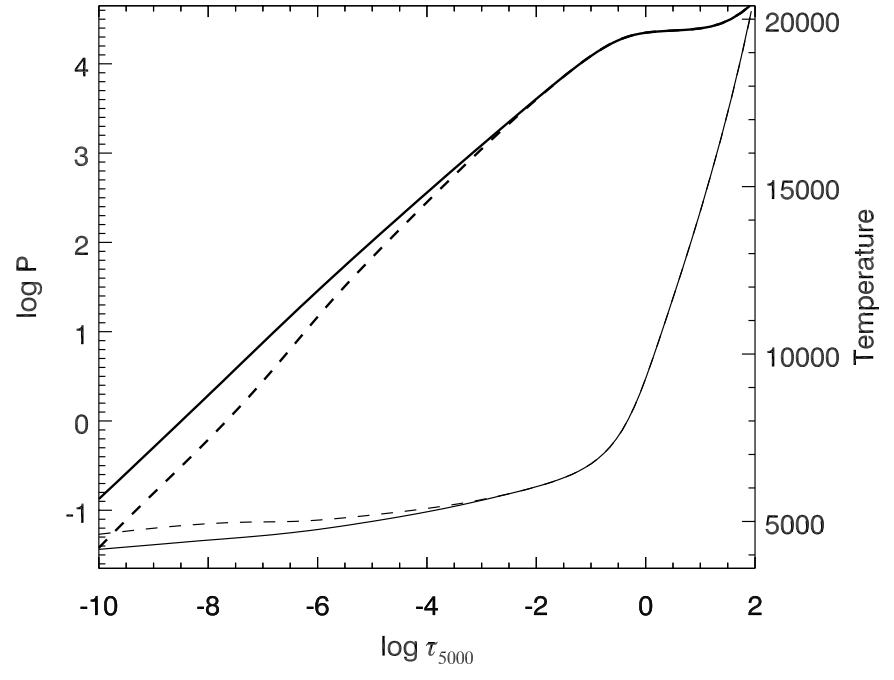

Fig. 1. Comparison between model atmospheres for $\gamma$ Equ calculated with two convergence criteria. Solid line: constant total flux and radiative equilibrium (see text). Dashed line: only constant total flux (as is standard in ATLAS9). The temperature gradient is shown by thin lines (right ordinate), the pressure gradient by thick lines (left ordinate).

an accurate continuum normalization in the orders which contain $\mathrm{H} \beta$ and $\mathrm{H} \alpha$ lines. Due to the extremely slow rotation of $\gamma$ Equ all spectra obtained between 1989 (ESO) and 1999 (SAAO) may be attributed to nearly the same rotation phase.

\section{Spectrum synthesis calculations with depth-dependent abundances}

Synthetic spectra of $\gamma$ Equ were calculated with a modified version of the SYNTHMAG code (Piskunov 1999). The main difference from the original version is the ability to specify individual sets of abundances for each layer in the model atmosphere. SYNTHMAG solves the equation for molecular equilibrium and determines the partial pressure of the line forming species, of protons and electrons. The resulting density of the stellar matter may be different from the original model structure but since hydrogen remains the dominant species, such deviations are negligible. The spectral synthesis is performed with the magnetic Feautrier algorithm for 7 limb angles and then convolved with the rotational velocity of the star.

Calculations of synthetic spectra with a magnetic field for $\gamma$ Equ but without abundance stratification are described in detail in PaperI. Here we mention only briefly the model atmosphere parameters $\left(T_{\text {eff }}=7700 \mathrm{~K}\right.$, $\log g=4.2,[\mathrm{M} / \mathrm{H}]=+0.5)$. The initial abundances and the model for approximating the magnetic field were taken from Paper II. Atomic parameters were extracted from VALD database (Piskunov et al. 1995; Ryabchikova et al. 1999; Kupka et al. 1999) and corrected, if necessary, by fitting the solar flux atlas (Kurucz et al. 1984).

We found it necessary to extend the $\gamma$ Equ model atmosphere calculations to very small optical depth. 
The standard ATLAS9 code by Kurucz (1993) starts calculations from the Rosseland optical depth $\log \tau_{\mathrm{R}}=-6.85$, while layers located as high as $\log \tau_{\mathrm{R}}=-9$ can still produce observable line strengths and line profile anomalies for overabundant elements. The usual convergence criterium (a constant integrated flux through all atmospheric layers) is not sensitive to temperature and pressure variations in optically very thin layers.

Therefore, we introduced an additional convergence criterium in our calculations which we defined via the radiative equilibrium equation:

$\int_{0}^{\infty} \kappa_{\nu} S_{\nu} \mathrm{d} \nu=\int_{0}^{\infty} \kappa_{\nu} J_{\nu} \mathrm{d} \nu$

where $S_{\nu}$ and $J_{\nu}$ are the source function and the mean intensity, $\kappa_{\nu}$ is the absorption coefficient. We start our ATLAS9 model calculations from $\log \tau_{R}=-10.0$ $\left(\log \tau_{5000} \approx-13\right)$ and continue the iterations till both criteria are satisfied within at least $1 \%$. This approach would not be valid for giants and supergiants, because of problems with balancing radiative and gas pressure, but it works well for Main Sequence stars and the models for $\gamma$ Equ needed not more than 31 iterations. Our final model consists of 99 layers. Of course, it can be considered only as a first approximation for the uppermost layers because of neglecting NLTE effects. Figure 1 shows a comparison between our 99 layer models calculated with the two convergence criteria: a constant total flux, as is standard in ATLAS9, and in addition a radiative equilibrium. Nota bene, both models provide the same energy distribution and $\mathrm{H} \alpha$ line profile.

\section{Observational evidence for abundance stratification}

Evidences for abundance stratification in the atmosphere of chemically peculiar stars are:

- core and wings of strong saturated lines are difficult to fit with a single abundance value;

- abundances obtained from high-excitation lines deviate significantly from the mean value;

- the microturbulence velocity parameter obtained in a standard abundance analysis is different for various elements;

- abundances obtained from lines of different ionization stages are discordant.

Individual discrepancies can be, of course, removed by modifying atmospheric parameters, but in some cases such a model does not even fit the observed energy distribution, photometric indices or hydrogen line profiles. One simply fails to find a unique homogeneous model atmosphere which removes all anomalies and hence we investigated abundance stratification as an explanation for these discrepancies.

In the spectrum of $\gamma$ Equ all four types of evidences seem to be significant. Starting from the diffusion profiles
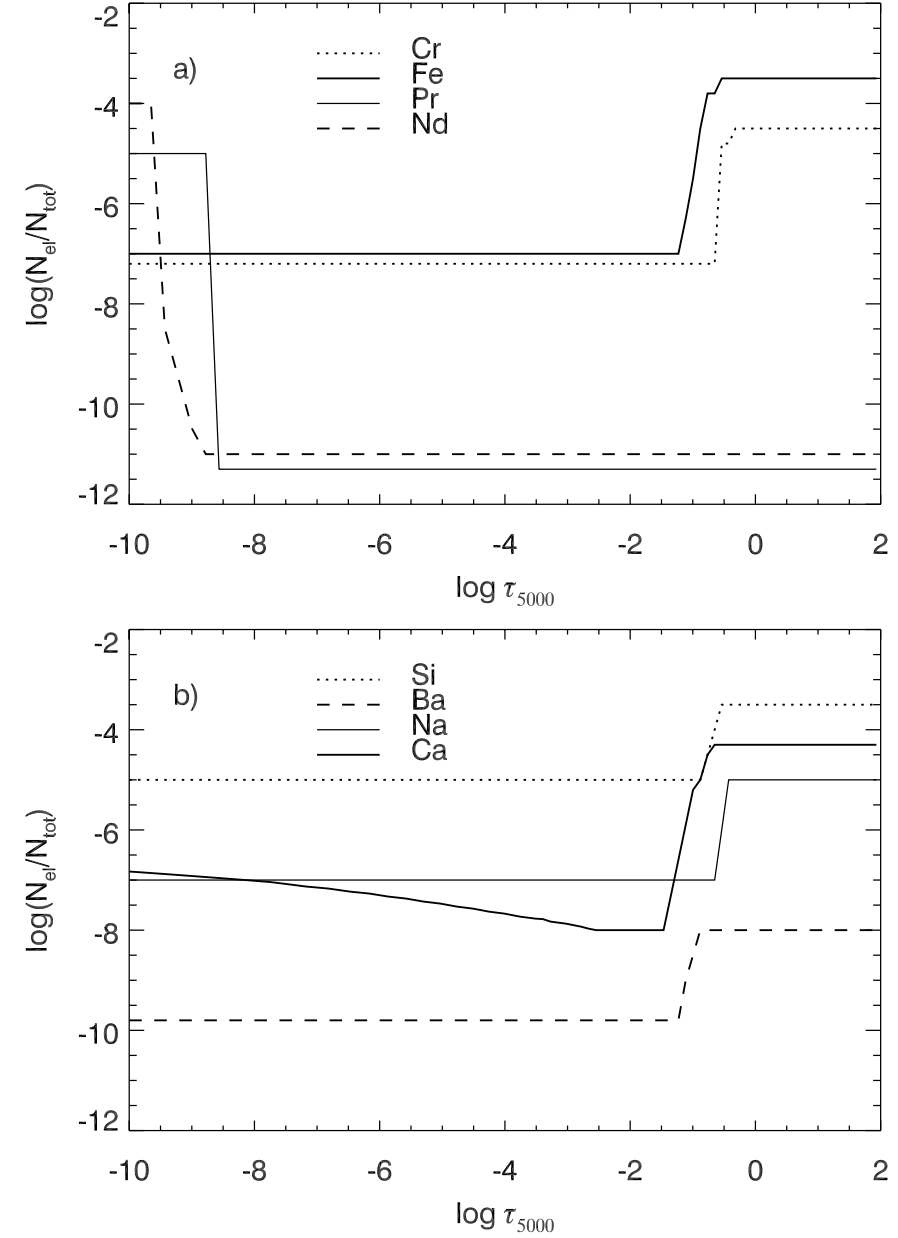

Fig. 2. Distribution of some chemical elements through the atmosphere of $\gamma$ Equ.

of Babel (1992) we derived by trial-and-error a model for an abundance stratification for various chemical elements and discuss them in the following. In any case we tried to fit the observations with the simplest possible model, a one-step distribution.

\subsection{Calcium}

26 spectral lines of $\mathrm{Ca}$ I and 8 lines of CaII in a large range of excitation potentials and oscillator strengths were studied in the spectrum of $\gamma$ Equ. The line list is given in Table 1 .

As a first step we used the vertical Ca abundance distribution derived by Babel (1992, see Fig. 3b of his paper). A small increase of the maximum abundance in deeper atmospheric layers was sufficient to describe all observed Ca lines reasonably well. The stratification profile is shown in Fig. $2 \mathrm{~b}$ as a thick solid line. Figures $3-6$ show further examples of spectral synthesis of the most interesting Ca lines. We chose these spectral regions also because they contain lines of other elements with indication for abundance stratification. Note that for the synthesis in the stratified atmosphere also other elements (discussed below and illustrated in Fig. 2) were considered to have a 
Table 1. A list of Ca lines, synthesized in the spectrum of $\gamma$ Equ with a depth-dependent abundance distribution. Asterisks mark the lines for which oscillator strengths corrections were applied. Oscillator strengths for Ca II lines marked by $\dagger$ are taken from TOPBASE (Seaton et al. 1992).

\begin{tabular}{lccccc}
\hline \multicolumn{1}{c}{$\lambda(\AA)$} & $\log g f$ & $E_{i}(\mathrm{eV})$ & $\lambda(\AA)$ & $\log g f$ & $E_{i}(\mathrm{eV})$ \\
\hline Ca I: & & & & & \\
4226.728 & 0.265 & 0.000 & 5601.277 & -0.552 & 2.525 \\
4283.011 & -0.292 & 1.886 & 5602.842 & -0.584 & 2.523 \\
5260.387 & -1.720 & 2.521 & 5604.939 & -0.765 & 4.763 \\
5261.704 & -0.591 & 2.521 & 6102.439 & -2.805 & 2.523 \\
5262.241 & -0.491 & 2.521 & 6102.723 & -0.862 & 1.879 \\
5264.237 & -0.575 & 2.523 & 6161.297 & -1.293 & 2.523 \\
5265.556 & -0.148 & 2.523 & 6162.173 & -0.167 & 1.898 \\
$5349.465^{*}$ & -0.178 & 2.709 & 6163.755 & -1.303 & 2.521 \\
5581.965 & -0.569 & 2.523 & 6717.681 & -0.596 & 2.709 \\
5588.749 & 0.313 & 2.525 & $6449.808^{*}$ & -0.415 & 2.521 \\
5590.114 & -0.596 & 2.521 & $6455.598^{*}$ & -1.357 & 2.523 \\
5594.462 & 0.051 & 2.523 & $6493.781^{*}$ & 0.219 & 2.521 \\
5598.480 & -0.134 & 2.521 & 6499.650 & -0.719 & 2.523 \\
\hline Ca II: & & & & & \\
3933.663 & 0.105 & 0.000 & 5285.266 & -1.272 & 7.505 \\
5001.479 & -0.517 & 7.505 & 5307.224 & -0.973 & 7.515 \\
5019.971 & -0.257 & 7.514 & $5339.188 \dagger$ & -0.079 & 8.438 \\
5021.138 & -1.217 & 7.514 & $6456.875 \dagger$ & 0.412 & 8.438 \\
\hline
\end{tabular}

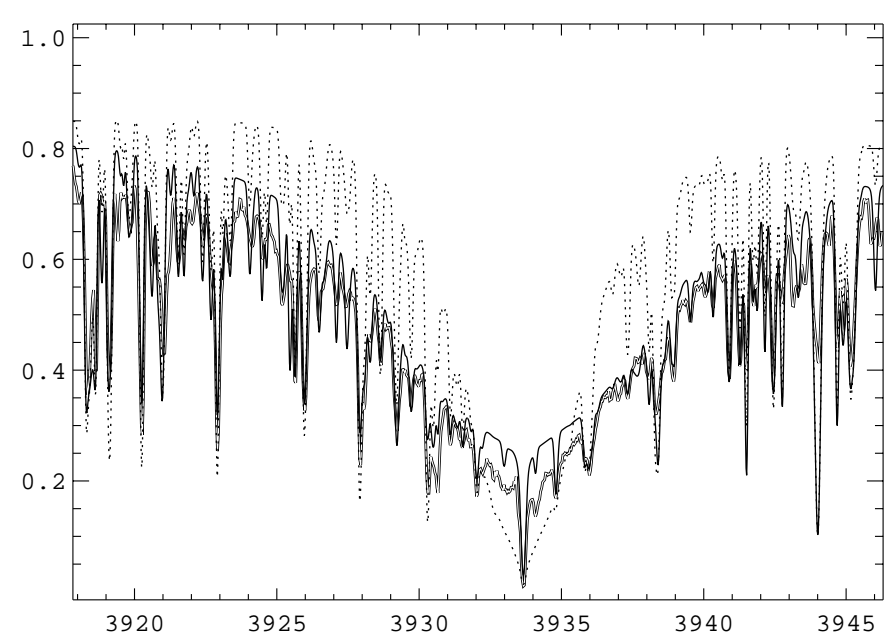

Fig. 3. Comparison between the synthetic spectra and observations for the Ca II K line at $3933.66 \AA$. The synthetic spectrum based on a stratified $\mathrm{Ca}$ abundance is shown with a solid line, while the dotted line corresponds to the calculations with the constant abundance of $\log \left(\mathrm{Ca} / N_{\text {tot }}\right)=-5.40$. Observations are indicated by a double line.

vertical abundance inhomogeneity, while the dotted lines in Figs. 3 to 12 represent synthetic spectra based on the homogeneous abundances taken from Paper II.

We did not try to improve further the agreement near the core of the CaII K line (Fig. 3), because it needs to include NLTE effects as well as to improve a modelling of

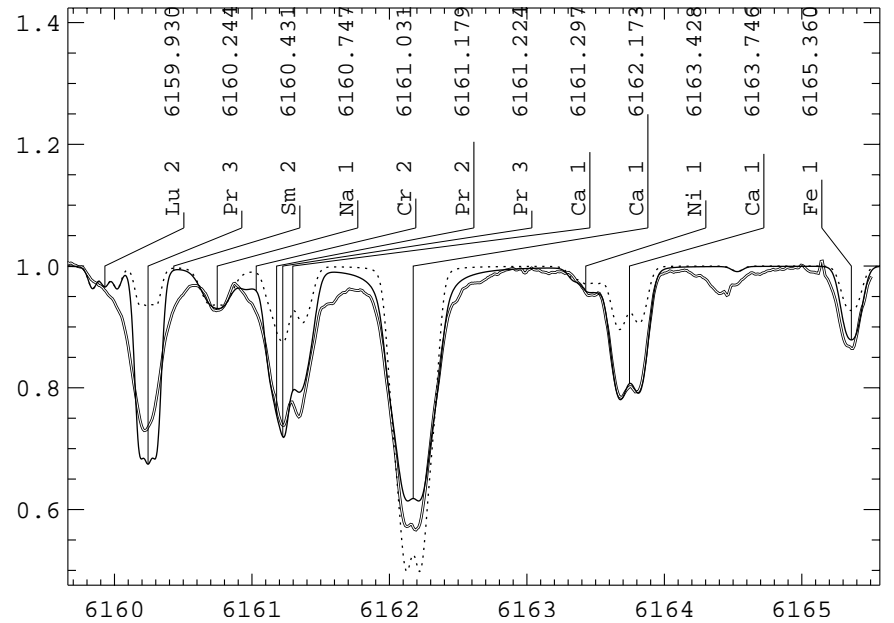

Fig. 4. The same as in Fig. 3 but for CaI lines in the 6160-6167 A spectral region. The dotted line represents the calculations with constant abundances of $\log \left(\mathrm{Ca} / N_{\text {tot }}\right)=$ $-5.40, \log \left(\mathrm{Na} / N_{\text {tot }}\right)=-5.73, \log \left(\mathrm{Fe} / N_{\text {tot }}\right)=-4.40$, and $\log \left(\operatorname{Pr} / N_{\text {tot }}\right)=-9.70$.

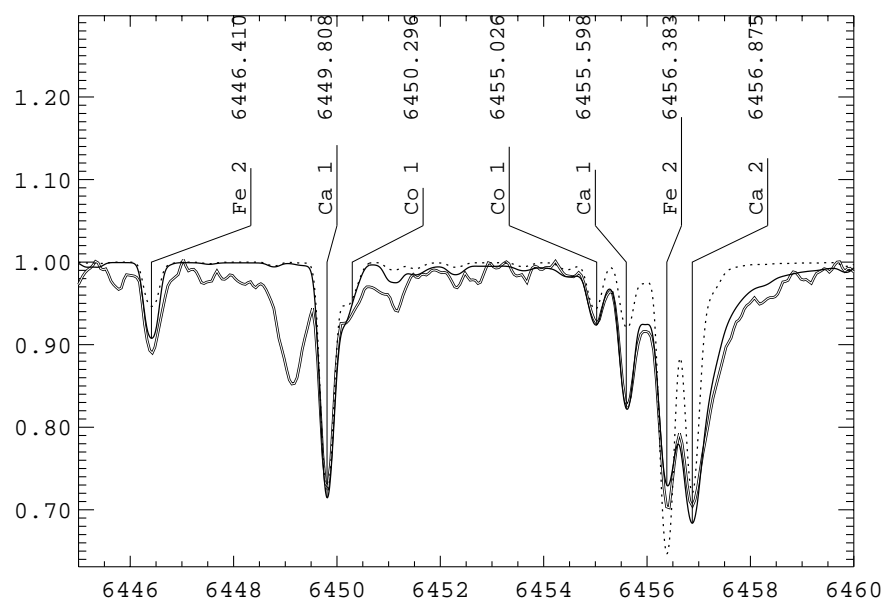

Fig. 5. The same as in Fig. 3, but for CaI and CaII lines in the 6446-6460 A spectral region.

the upper atmospheric layers taking into account abundance stratification. A stratified Ca abundance in the atmosphere of $\gamma$ Equ significantly improves the fit of the synthetic spectrum to observations in the entire spectral region available to us, ranging from 3900 to $8000 \AA$. We refer to the Ca I $\lambda \lambda 6162.173,6163.746,6166.439,6169.042$ and $6169.563 \AA$ lines (some are shown in Fig. 4) which differ in oscillator strengths, low level excitation energies ( $E_{i}=1.9 \mathrm{eV}$ for the first line and $E_{i}=2.52 \mathrm{eV}$ for the other lines), and which are obviously formed in different atmospheric levels. To fit reasonably well all line profiles with a constant $\mathrm{Ca}$ abundance is only possible with an effective temperature increased by more than $1000 \mathrm{~K}$, which contradicts the observed photometry, energy distribution and hydrogen line profiles. The first two lines do not show any radial velocity changes larger than $30 \mathrm{~m} \mathrm{~s}^{-1}$ according to Paper I. A possible depth dependence of $R V$ amplitudes accross the atmosphere of $\gamma$ Equ due to pulsation will be discussed in Sect. 5 . 


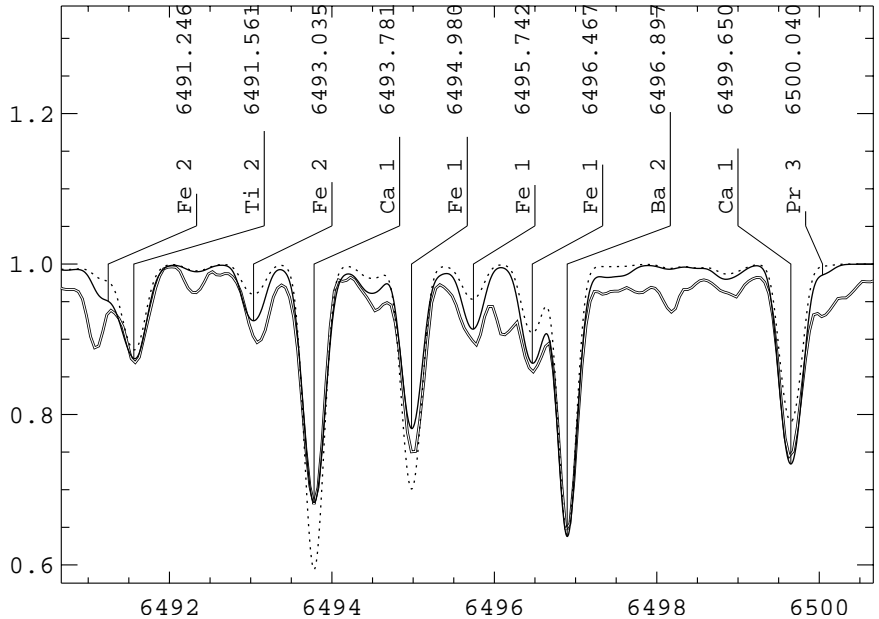

Fig. 6. The same as in Fig. 3, but for Ca I lines in the 6490$6500 \AA$ spectral region.

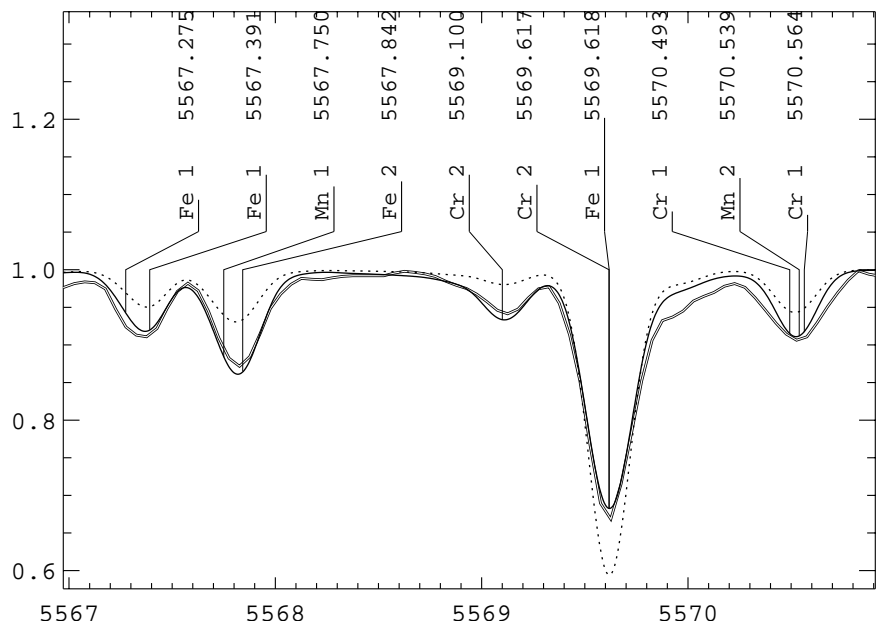

Fig. 7. The same as in Fig. 3, but for Cr II lines in the 5667$5571 \AA$ spectral region. The synthetic spectrum of the homogeneous atmosphere is based on $\log \left(\mathrm{Cr} / N_{\text {tot }}\right)=-5.43$.

The Ca abundance stratification derived by us agrees very well with the expected stratification in stellar atmospheres due to diffusion with a weak mass loss of $\approx 2.5 \times 10^{-15} M_{\odot} \mathrm{yr}^{-1}$. This distribution is similar to that obtained by Babel $(1992)$ for a hotter $\left(T_{\text {eff }}=8500 \mathrm{~K}\right) \mathrm{mag}$ netic star, $53 \mathrm{Cam}$, and by Wade et al. (2001) for $\beta \mathrm{CrB}$. As it was pointed out by Babel (1994) abundance stratification of Ca seems to be typical for magnetic stars with $T_{\text {eff }}<9000 \mathrm{~K}$.

\subsection{Iron-peak elements}

The most significant indicator of $\mathrm{Cr}$ and $\mathrm{Fe}$ abundance stratification in $\gamma$ Equ is an increased intensity of $\mathrm{Cr} I \mathrm{II}$ and $\mathrm{Fe}$ II lines with $E_{i}>10 \mathrm{eV}$ in comparison with lines with $E_{i} \approx 3-4 \mathrm{eV}$. Uncertainty of the oscillator strengths used for high-excitation lines could not account for this anomaly, because the high-excitation Fe II lines provide the same abundance in the spectrum of the normal star HD 32115 (Ryabchikova, private communication) as do all

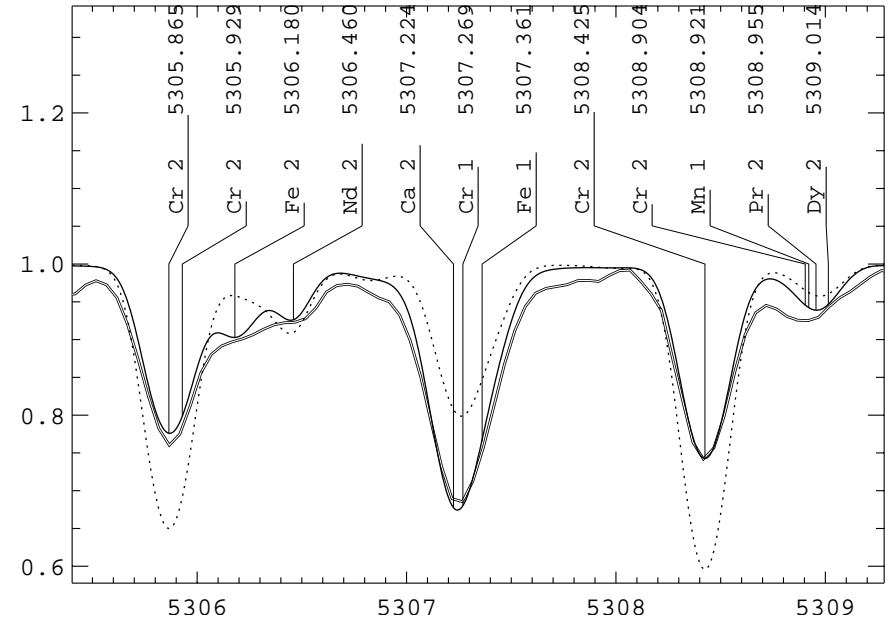

Fig. 8. The same as in Figs. 3 and 7, but for Cr II lines in the 5305-5309 A spectral region.

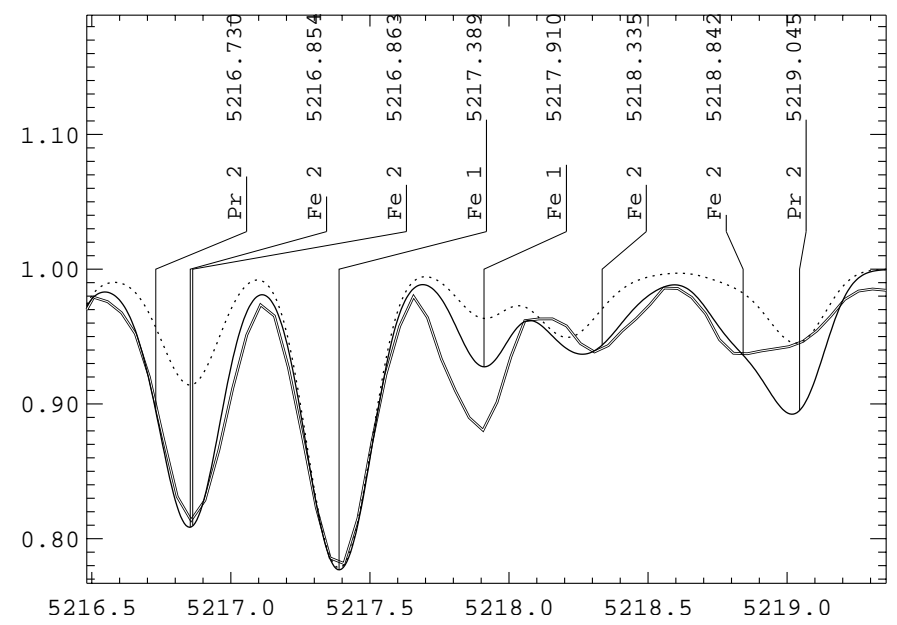

Fig. 9. The same as in Fig. 3, but for Fe lines in the 5216$5219 \AA$ spectral region. The synthetic spectrum of the homogeneous atmosphere is based on $\log \left(\mathrm{Fe} / N_{\text {tot }}\right)=-4.40$.

other Fe lines. Similar to Ca we started our calculations with the Cr diffusion profiles from Babel (1992) and varied lower and higher abundances and the optical depth of the abundance jump until we got satisfactory fits for the majority of strong and weak $\mathrm{Cr}$ and Fe lines of different excitation potentials. Comparisons of our synthetic spectra with observations are presented for Cr II $\lambda 5569.11 \AA\left(E_{i}=\right.$ $10.87 \mathrm{eV})$ in Fig. 7, for Cr II $\lambda \lambda 5305.865,5308.425 \AA$ $\left(E_{i}=4.07 \mathrm{eV}\right)$ in Fig. 8, and for CrI $\lambda 5296.691 \AA$ $\left(E_{i}=0.98 \mathrm{eV}\right)$ in Fig. 13. Similar comparisons for Fe lines are presented in Figs. 9, 13, and 14. While the Fe I line at $\lambda 5217.389 \AA\left(E_{i}=3.21 \mathrm{eV}\right)$ and the Fe II lines at $\lambda \lambda 6147.741,6149.258 \AA\left(E_{i}=3.89 \mathrm{eV}\right)$ are reasonably well fitted with a homogeneous Fe abundance, this is not possible for the high-excitation Fe II lines at $\lambda \lambda$ 5216.854, $5216.863,5291.666 \AA\left(E_{i}=10.5 \mathrm{eV}\right)$. Cr and Fe abundances for the non-stratified atmosphere are taken from Paper II.

The figures clearly show a significant improvement of all synthesized $\mathrm{Cr}$ and $\mathrm{Fe}$ lines for a model atmosphere 


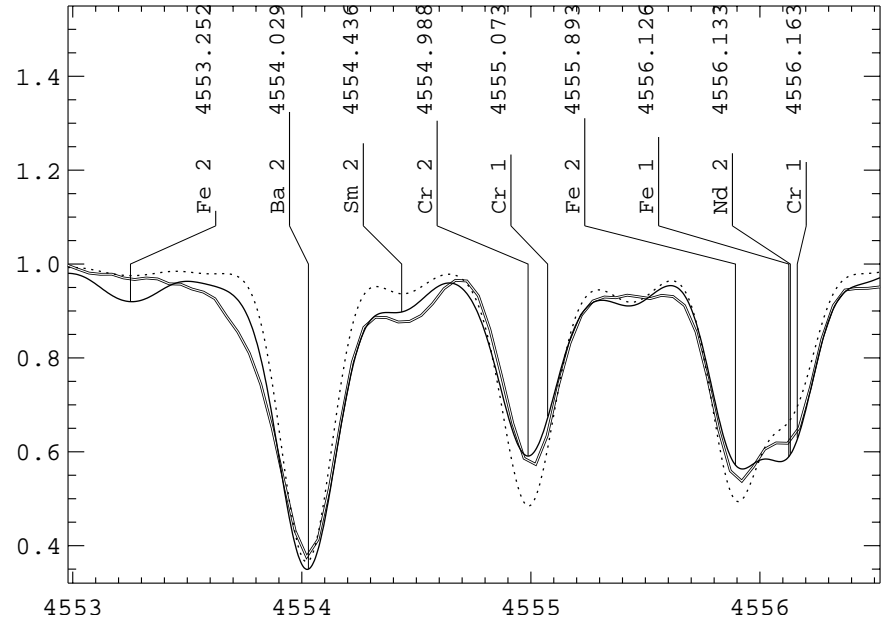

Fig. 10. The same as in Fig. 3 , but for Ba II at $\lambda 4554.03 \AA$. The synthetic spectrum of the homogeneous atmosphere is based on $\log \left(\mathrm{Ba} / N_{\text {tot }}\right)=-9.06$.

with stratified abundance distributions. Changing $T_{\text {eff }}$ cannot help to achieve the same agreement. In the case of $\mathrm{Cr}$ the effective temperature of the star needs to be increased by at least $800 \mathrm{~K}$ to achieve consistent abundances for Cr II lines with different excitation energy. But with such an adjustment for the temperature it is impossible to achieve ionization equilibrium for the entire set of Cr I and Cr II covering the large range of excitation energy.

Figure 2 (upper panel) illustrates the abundance stratification for $\mathrm{Cr}$ and $\mathrm{Fe}$ as we derived it from our spectroscopic analysis.

Our abundance stratification for chromium $\left(\left[\mathrm{Cr}_{\min }\right]=\right.$ $\left.-7.2,\left[\mathrm{Cr}_{\max }\right]=-4.5, \log \tau_{0}=-0.6\right)$ and for iron $\left(\left[\mathrm{Fe}_{\min }\right]=-7.0,\left[\mathrm{Fe}_{\max }\right]=-3.5, \log \tau_{0}=-1.0\right)$, where $\log \tau_{0}$ indicates the optical depth of the abundance jump, agree fairly well with the results obtained for $53 \mathrm{Cam}$ (Babel 1992; Babel \& Lanz 1992) and $\beta$ CrB (Wade et al. 2001):

53 Cam: $\left[\mathrm{Cr}_{\min }\right]=-6.5,\left[\mathrm{Cr}_{\max }\right]=-3.7, \log \tau_{0}=-0.9$, and $\left[\mathrm{Fe}_{\min }\right]=-5.3,\left[\mathrm{Fe}_{\max }\right]=-2.7, \log \tau_{0}=-0.9$;

$\beta \mathrm{CrB}:\left[\mathrm{Cr}_{\min }\right]=-6.4,\left[\mathrm{Cr}_{\max }\right]=-3.6, \log \tau_{0}=-0.6$, and $\left[\mathrm{Fe}_{\min }\right]=-5.8,\left[\mathrm{Fe}_{\max }\right]=-2.6, \log \tau_{0}=-0.7$.

It is interesting to note that the abundance difference between the inner and outer layers is the same for all three stars while the optical depth where this change occurs is lower in the roAp star $\gamma$ Equ than in the non-pulsating stars 53 Cam and $\beta$ CrB.

We also tried to deduce the abundance stratification of $\mathrm{Mn}$ from the following lines: Mn I $\lambda \lambda 5394.674$ $\left(E_{i}=0.0 \mathrm{eV}\right), 6013.498 \quad\left(E_{i}=3.06 \mathrm{eV}\right), \quad 5399.489$ $\left(E_{i}=3.84 \mathrm{eV}\right)$, Mn II $\lambda \lambda 4283.766\left(E_{i}=5.35 \mathrm{eV}\right)$, and $5299.386\left(E_{i}=9.82 \mathrm{eV}\right)$. No clear evidence for a stratification pattern similar to $\mathrm{Cr}$ and Fe could be found. We derived an upper limit for the abundance difference (with a consequently rather inaccurate optical depth for the jump) to $\left[\mathrm{Mn}_{\min }\right]=-6.3,\left[\mathrm{Mn}_{\max }\right]=-5.3, \log \tau_{0}=-0.65$.

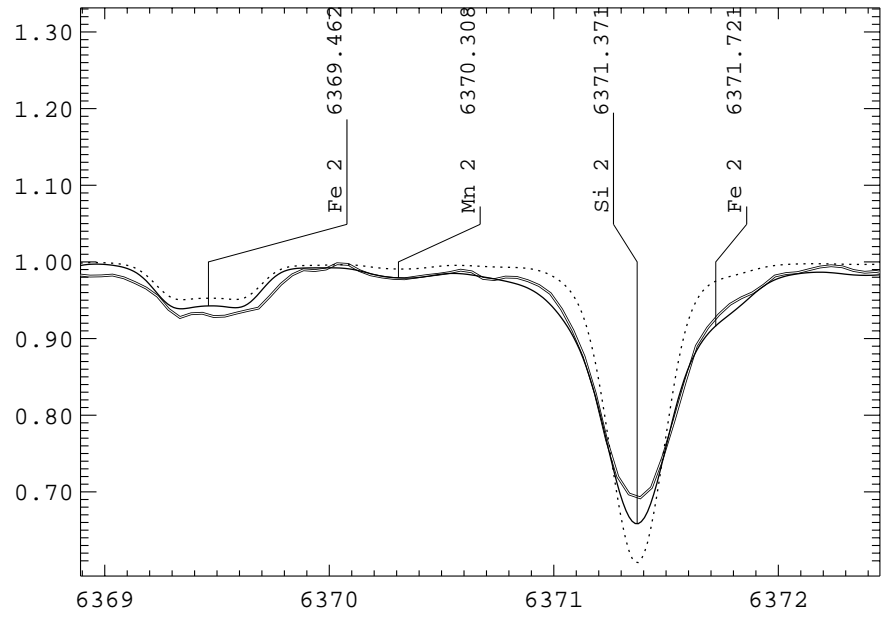

Fig. 11. The same as in Fig. 3, but for Si II at $\lambda 6371.37 \AA$. The synthetic spectrum of the homogeneous atmosphere is based on $\log \left(\mathrm{Si} / N_{\text {tot }}\right)=-4.42$.

\section{3. $\mathrm{Ba}, \mathrm{Si}$, and $\mathrm{Na}$}

A few strong lines of Ba II are observable in the spectrum of $\gamma$ Equ. Different abundances are required to fit the core and wings of each line, if stratification is present. And indeed, a synthetic spectrum calculated with the homogeneous abundance of $\log \left(\mathrm{Ba} / N_{\text {tot }}\right)=-9.06$, obtained in Paper II, fits well the line-core, but not the wing (Fig. 6, dotted line, Ba II $\lambda 6496.897)$. These anomalously wide wings, however, can be fitted with a higher $\mathrm{Ba}$ abundance in deeper atmospheric layers. Figure 10 shows a comparison of the observed resonance line Ba II $\lambda 4554.03$ (double line in in the figure) with two types of synthetic spectra. Both calculations used hyperfine splitting constants from Biehl (1976). The synthetic spectrum without stratification (abundances were taken from Paper II) is plotted with a dotted line, whereas the thin line is based on the following abundance stratification: $\left[\mathrm{Ba}_{\min }\right]=-9.8$, $\left[\mathrm{Ba}_{\max }\right]=-8.0, \log \tau_{0}=-1.0$. This stratification profile is shown in the lower panel of Fig. 2 as dashed line and it provides a reasonably good fit to the observed line profiles of all strong Ba II lines (see also Figs. 6 and 14).

The situation is similar for silicon and our analysis has to be based on cores and wings of the strong Si II $\lambda \lambda$ 5056, 6347 and $6371 \AA$ lines. We obtained the following abundance stratification $\left[\mathrm{Si}_{\min }\right]=-5.3,\left[\mathrm{Si}_{\max }\right]=-3.5$, $\log \tau_{0}=-0.7$ which is shown in the lower panel of Fig. 2 as dotted line. The neutral silicon lines are more of a problem, because their atomic parameters are inaccurately known. For example, the Si I lines of multiplets 29 and 30 (6130-6160 A) required a correction for the oscillator strengths of about -0.5 dex to fit these lines to the solar spectrum. A comparison between observations and synthetic spectra calculated for stratified and non-stratified atmospheres is shown for Si II $\lambda 6371.37$ in Fig. 11. Again, the mean abundance of $\log \left(\mathrm{Si} / N_{\text {tot }}\right)=-4.42$ in the nonstratified atmosphere was taken from Paper II. Stratified $\mathrm{Ba}$ and $\mathrm{Si}$ abundances improved also significantly the 


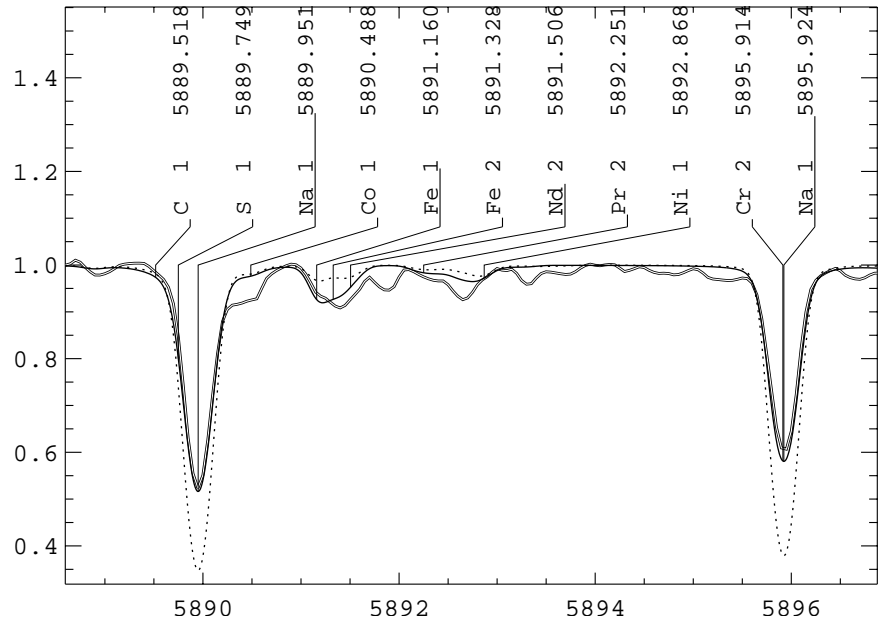

Fig. 12. The same as in Fig. 3, but for Na I in the 5889-5896 spectral region. The synthetic spectrum of the homogeneous atmosphere is based on $\log \left(\mathrm{Na} / N_{\text {tot }}\right)=-5.73$.

fit for BaII-Si I lines in the $6140 \AA$ spectral region (see Fig. 14).

Neutral sodium has strong resonance lines at $\lambda \lambda$ 5889.95, 5895.92 $\AA$ and a few weaker lines with $E_{i}=2.10 \mathrm{eV}$. Usually, resonance lines are avoided in abundance analyses due to possible NLTE effects. Ryabchikova et al. (Paper II) derived $\log \left(\mathrm{Na} / N_{\text {tot }}\right)=-5.27$ based on 3 weak lines of multiplets 11 and 12 , but the strongest line among them at $4668.56 \AA$ provided an abundance closer to the solar value. To improve the statistics for a new analysis of the $\mathrm{Na}$ abundance we decided to include also lines more to the red $(\lambda \lambda 5682.63,6154.23$ and $6160.75 \AA$ ) and with a lower quality ranking in VALD than we were usually willing to accept. A new mean abundance was derived as $\log \left(\mathrm{Na} / N_{\text {tot }}\right)=-5.73 \pm 0.20$ and hence it seems that the $\mathrm{Na}$ abundance has been overestimated in Paper II. But one needs even smaller than solar Na abundances to fit the cores of resonance lines. The abundance stratification for sodium derived from all the lines mentioned is $\left[\mathrm{Na}_{\text {min }}\right]=-7.0,\left[\mathrm{Na}_{\text {max }}\right]=-5.0, \log \tau_{0}=-0.54$ and is shown in the lower panel of Fig. 2 as thin solid line. This stratification profile allows to fit reasonably well all $\mathrm{Na}$ I lines, including even the resonance lines (see Figs. 12 and 4).

\section{4. $\operatorname{Pr}$ and Nd}

All elements discussed up to now are concentrated below $\log \tau_{5000}=-1.0$ and have above this optical depth solar or even less than solar abundances. Rare earth elements (REE) show an opposite behaviour. Ryabchikova et al. (2001) performed detailed investigations of $\mathrm{Pr}$ and $\mathrm{Nd}$ lines in the atmospheres of roAp stars including $\gamma$ Equ. They found for all these stars much stronger PrIII and Nd III lines than would be expected from an analysis of the lines of the first ions. The suspicion that this effect might be caused by incorrect atomic data is contradicted by the fact that a similar analysis of non-pulsating Ap stars with

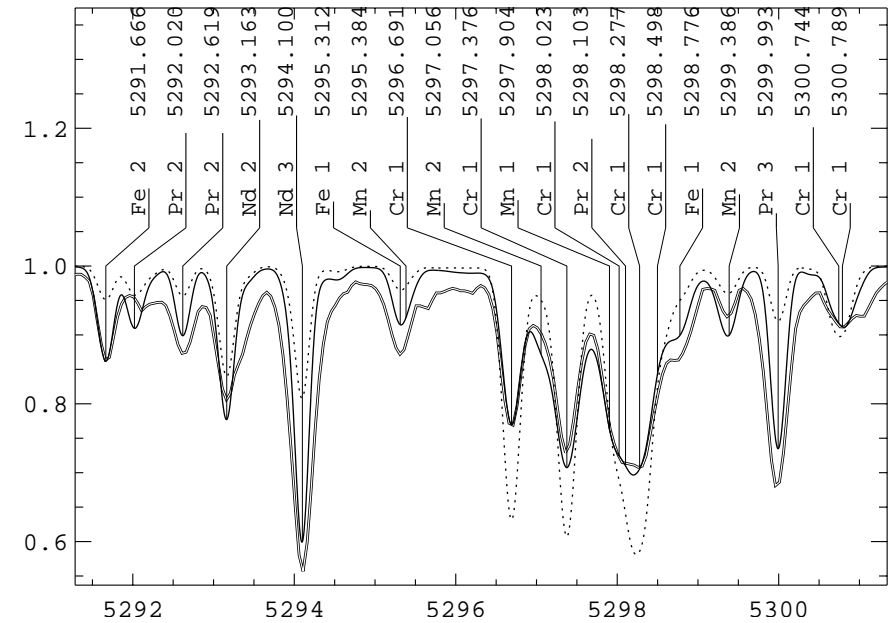

Fig. 13. The same as in Fig. 3, but for Nd III at $\lambda 5294.10 \AA$ and Pr III at $\lambda 5299.99 \AA$. The synthetic spectrum of the homogeneous atmosphere is based on $\log \left(\operatorname{Pr} / N_{\text {tot }}\right)=-9.70$ and $\log \left(\mathrm{Nd} / N_{\text {tot }}\right)=-9.00$.

otherwise similar properties do not show this imbalance. For $\gamma$ Equ we observe on average an abundance difference of 1.5 dex between lines of the first and second ions. To explain this REE anomaly the existence of an overabundant layer of REE in the upermost atmosphere was first proposed by Ryabchikova et al. (2000) in an abundance study of the cooler roAp stars 10 Aql and HD 122970.

According to the Saha equation the second ions of Pr and Nd are dominant in LTE atmosphere of $\gamma$ Equ below $\log \tau_{5000}=-0.5$ (high temperature) and above $\log \tau_{5000}=-8.0$ (low density). The observed Pr III and Nd III lines with low excitation energies are very strong and hence cannot originate in enriched deep atmospheric layers. The only possibility to obtain strong lines of the second ions and at the same time much weaker lines of the first ions is to postulate a thin layer above $\log \tau_{5000}=-8$ with a large overabundance of REE. At these optical depths, however, one cannot ignore NLTE effects and therefore all our LTE abundance estimates have to be considered as preliminary. However, in this paper we want to develop only a qualitative scenario for the REE abundance stratification in the atmosphere of $\gamma$ Equ.

Oscillator strengths and Landé factors for Nd III lines were taken from Bord (2000), and for Pr III lines from Bord (private communication). The reliability of atomic data was checked in a comparative abundance study of pulsating and non-pulsating Ap and Am stars (Ryabchikova et al. 2001). Here we mainly considered $6139-6165 \AA$ spectral region, which was observed with $R=166000$ and contains strong Nd III $\lambda 6145.07$ and Pr III $\lambda 6160.24$ lines. These spectral features are additionally broadened due to pulsation (see Paper I). Consequently, our abundance analysis was based rather on fitting equivalent widths than on line profiles. The abundance stratification which allowed us to fit simultaneously strong and weak lines of second and first ions is shown in the upper panel of Fig. 2 with a thin solid line $(\mathrm{Pr})$ and a thick dashed line $(\mathrm{Nd})$ and is 


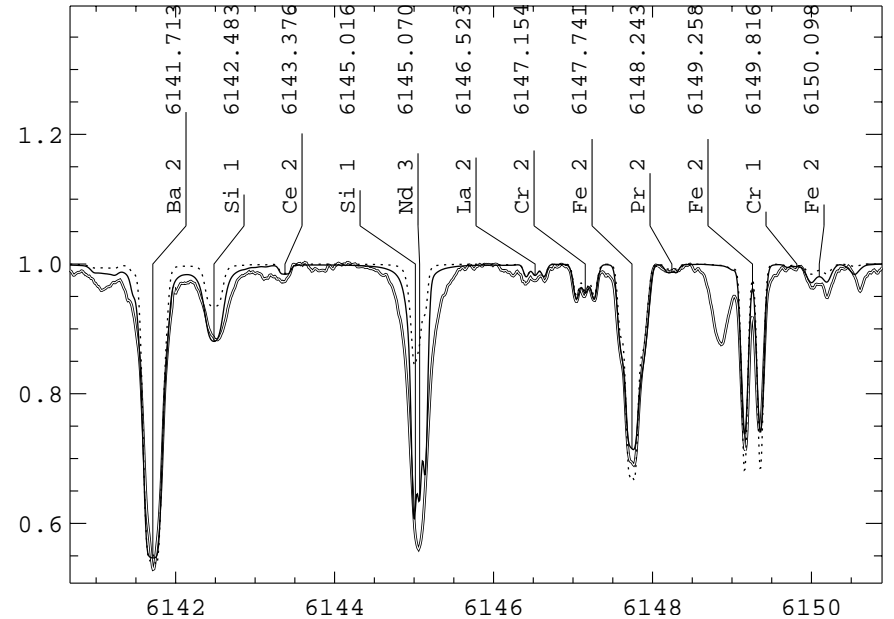

Fig. 14. The same as in Fig. 13, but for Nd III at $\lambda 6145.07 \AA$.

given by: $\left[\mathrm{Pr}_{\min }\right]=-11.3,\left[\mathrm{Pr}_{\max }\right]=-5.0, \log \tau_{0}=-8.5$, and $\left[\mathrm{Nd}_{\text {min }}\right]=-11.0,\left[\mathrm{Nd}_{\max }\right]=-4.0, \log \tau_{0}=-9.5$. Even with the increased abundance above $\tau_{0}$ some of the Pr III and Nd III lines are still too weak in the synthetic spectrum. A deeper optical depth of the abundance jump would cause too strong lines of the first ions. Our best compromise is illustrated for Pr III lines in Figs. 4 and 13, and for Nd III lines in Figs. 13 and 14. The synthetic spectra plotted with a dotted line are computed for nonstratified Pr and Nd abundances which were slightly improved over the values published in Ryabchikova et al. $\left(2001 ; \log \left(\operatorname{Pr} / N_{\text {tot }}\right)=-9.84\right.$ and $\left.\log \left(\mathrm{Nd} / N_{\text {tot }}\right)=-8.95\right)$ to better fit lines in the red spectral region.

No diffusion calculations exist for REE elements and therefore it is not possible to compare our results directly with predictions. However, diffusion calculations for $\mathrm{Hg}$ in hotter stars (Michaud et al. 1974) indicate large Hg overabundances in the uppermost atmospheric layers, where $N(\mathrm{Hg}$ II $) \approx N(\mathrm{Hg}$ III $)$ and gravitational forces equal radiative forces. It may be speculated that we observe in $\gamma$ Equ a similar effect for REE.

\section{The pulsating atmosphere of $\gamma$ Equ}

A spectroscopic sequence with high time and spectral resolution provided us with information about the depth dependence of the pulsation velocity field of $\gamma \mathrm{Equ}$ in the $\lambda 6139$ to $6165 \AA$ spectrum region (see Paper I). As is discussed in this reference, the $R V$ amplitudes were different for spectral lines of different elements and ionisation stages and - for obvious reasons - could be best determined for rather strong lines of which some are listed in the following Table 2. The last two columns contain estimates of formation depths, based on contribution functions, for line cores in non-stratified and in stratified atmospheres. These depths were calculated according to the definition of the contribution function similar to that introduced by Achmad et al. (1991). Because of the large optical depths range in question for $\gamma$ Equ we calculated
Table 2. Radial velocity amplitudes and optical depths of linecore forming regions for some spectral lines of $\gamma$ Equ.

\begin{tabular}{lrrcc}
\hline Ion & $\lambda$ & $\Delta R V$ & \multicolumn{2}{c}{$\log \tau_{5000}$} \\
& \multicolumn{1}{c}{$\AA$} & \multicolumn{1}{c}{$\mathrm{m} \mathrm{s}^{-1}$} & non-stratified & stratified \\
\hline Nd III & 6145.07 & $470(21)$ & -3.4 & -8.3 \\
Pr III & 6160.24 & $788(37)$ & -2.9 & -7.0 \\
Ba II & 6141.71 & $93(16)$ & -4.2 & -3.0 \\
Ca I & 6162.17 & $\leq 30$ & -2.7 & -1.1 \\
Ca I & 6163.76 & $\leq 35$ & -1.3 & -0.6 \\
\hline
\end{tabular}

the mean formation depth for a given spectral line as a logarithmic mean.

It is difficult to interprete the results of Table 2 in the framework of a chemically uniform atmosphere. Spectral lines which would be formed at nearly the same optical depths have $R V$ amplitudes which differ by a factor of 5 to 8 ! However, a spotty atmospheric structure could explain part of this phenomenon. Calcium, e.g., could be concentrated in a spot (or spots) which happen(s) to coincide with a pulsation node.

In a stratified atmosphere both REE lines are formed in the uppermost atmospheric layers, the Ba II line is formed in intermediate layers and the CaI lines are formed much deeper. Hence, we seem to observe an increase of pulsation amplitudes towards the outer atmosphere. This model also explains the smaller - but nevertheless detectable $R V$ amplitudes derived from the lines of first REE ions (see Paper I) which are formed deeper in a stratified atmosphere than doubly ionized REE lines. Our stratification model, as is illustrated in Fig. 2, could also account for observed phase shifts in radial velocities of singly relative to doubly ionized REE lines (see Paper I).

We mentioned in Sect. 4.4 that NLTE effects should be considered. Among the REE such NLTE calculations are available only for $\mathrm{Eu}$ (Mashonkina et al. 2002). The size of NLTE effects in an atmosphere with $T_{\text {eff }}=7750 \mathrm{~K}$ for Eu grows towards the upper layers. We expect that NLTE effects will result for $\mathrm{Pr}$ and $\mathrm{Nd}$ in a smaller abundance jump and/or a shift of the abundance gradient to slightly deeper layers in the atmosphere.

The rather large $R V$ amplitudes of $\approx 350 \mathrm{~ms}^{-1}$ and phase shifts observed for weak NaI lines (see Paper I) are difficult to interprete in the framework of our present stratified model atmosphere. However, the NaI $\lambda 6160.75 \AA$ line is situated in the overlapping wings of strong PrII and PrIII lines (see Fig. 4) and the large pulsation amplitude of the latter may likely influence the $\mathrm{Na}$ I line measurements. But this explanation would not work for another $\mathrm{NaI} \lambda 6154.23 \AA$ line with no such blending REE components and a $\triangle R V$ similar to the $6160.75 \AA$ line. Considering the stratification profile we have determined for $\mathrm{Na}$ (Fig. 2), both lines form below $\log \tau_{5000}=-1.0$, where we also expect the Ca lines to be formed, but which have a much smaller $R V$ amplitude. $\mathrm{Na}$ spots located differently to those of Ca may provide an "ad hoc", but admittedly unsatisfying way-out of this 
discrepancy. Spectroscopic time series in the region of Na I resonance lines could be very helpful for clarifying this problem.

\section{Conclusion}

Abundance stratification in the atmosphere of the rapidly oscillating Ap star $\gamma$ Equ is evident from high quality spectra. Line wings and cores as well as lines of the same ion but with different excitation potential can only be fitted at the same time with an assumed abundance stratification in the model atmosphere. Furthermore, a discrepancy of abundances obtained from lines of the same element but different ionization stages and found for homogeneous atmospheres can be eliminated with a stratification model.

$\mathrm{Ca}, \mathrm{Cr}, \mathrm{Fe}, \mathrm{Ba}, \mathrm{Si}$, and $\mathrm{Na}$ seem to be overabundant in deeper atmospheric layers, but normal to underabundant in the upper layers with a transition in the typical line forming region of $-1.5<\log \tau_{5000}<-0.5$. Pr and $\mathrm{Nd}$ from the group of REE have an opposite profile. Their abundance is more than 6 dex higher, above $\log \tau_{5000} \approx-8.0$, than in the deeper atmospheric layers.

According to Kochukhov \& Ryabchikova (2001) the $R V$ amplitudes of REE lines are 5 to 8 times larger than for, e.g., $\mathrm{Ca}$ and $\mathrm{Ba}$, which could be explained as a consequence of a stratified atmosphere. Within the context of this model, doubly ionized REE lines are formed in the uppermost layers, whereas the lines of first REE ions are formed deeper in a stratified atmosphere. This depth dependence would explain the considerably larger $R V$ amplitudes observed for lines of the second relative to those of the first ions. However, we have to remember the difficulty in understanding within the framework of the same model the behaviour of $\mathrm{Na}$ lines.

Acknowledgements. This work was supported by the Fonds zur Förderung der wissenschaftlichen Forschung (projects P14984 and $P 11882-P H Y$ ) and by the Swedish Naturvetenskapliga forskningsrådet. T.R. also thanks the Russian National Program "Astronomy" (grant 1.4.1.5) and the RFBR (grant 00-15-96722) for partial funding.

\section{References}

Achmad, I., de Jager, C., \& Nieuwenhuijzen, H. 1991, A\&A, 250,445

Babel, J. 1992, A\&A, 258, 449

Babel, J. 1994, A\&A, 283, 189

Babel, J., \& Lanz, T. 1992, A\&A, 263, 232

Baldry, I. K., Bedding, T. R., Viskum, M., Kjeldsen, H., \& Frandsen, S. 1998, MNRAS, 295, 33
Baldry, I. K., Kurtz, D. W., \& Bedding, T. R. 1998, MNRAS, 300, L39

Baldry, I. K., Viskum, M., Bedding, T. R., Kjeldsen, H., \& Frandsen, S. 1999, MNRAS, 302, 381

Baldry, I. K., \& Bedding, T. R. 2000, MNRAS, 318, 341

Biehl, D. 1976, Ph.D. Thesis, Christian-Albrechts-Universität, Kiel, Institute für Theoretische Physik und Sternwarte

Bord, D. 2000, A\&AS, 144, 517

Cowley, C. R., \& Bord, D. J. 1998, in The Scientific Impact of the Goddard High Resolution Spectrograph, ed. J. C. Brandt, T. B. Ake, \& C. C. Peterson, ASP Conf. Ser., 143, 346

Kanaan, A., \& Hatzes, A. P. 1998, ApJ, 503, 848

Kochukhov, O., \& Ryabchikova, T. 2001, A\&A, 374, 615, Paper I

Kupka, F., Piskunov, N., Ryabchikova, T. A., Stempels, H. C., \& Weiss, W. W. 1999, A\&AS, 138, 119

Kurtz, D. W. 1982, MNRAS, 200, 807

Kurtz, D. W., \& Wegner, G. 1979, ApJ, 232, 510

Kurucz, R. L. 1993, CDROM13, SAO, Cambridge

Kurucz, R. L., Furenlid, I., Brault, J., \& Testerman, L. 1984, NSO Atlas No. 1: Solar Flux Atlas from 296 to 1300 nm, Sunspot, NSO

Libbrecht, K. G. 1988, ApJ, 330, L51

Malanushenko, V., Savanov, I., \& Ryabchikova, T. 1998, IBVS, No. 4650

Martinez, P., Weiss, W. W., Nelson, M. J., et al. 1996, MNRAS, 282,243

Mashonkina, L. I., Ryabtsev, A. N., \& Ryabchikova, T. A. 2002, Astron. Lett., 28, 41

Michaud, G. 1970, ApJ, 160, 641

Michaud, G., Reeves, H., \& Charland, Y. 1974, A\&A, 37, 313

Piskunov, N., Kupka, F., Ryabchikova, T. A., Weiss, W. W., \& Jeffery, C. S. 1995, A\&AS, 112, 525

Piskunov, N. E. 1999, in 2nd Workshop on Solar Polarization, ed. J. Stenflo, \& K. N. Nagendra (Kluwer Academic Publishers, Dodrecht), 515

Ryabchikova, T. A., Adelman, S. J., Weiss, W. W., \& Kuschnig, R. 1997, A\&A, 322, 234, Paper II

Ryabchikova, T. A., Piskunov, N. E., Stempels, H. C., Kupka, F., \& Weiss, W. W. 1999, Phys. Scr., T83, 162

Ryabchikova, T. A., Savanov, I. S., Hatzes, A. P., Weiss, W. W., \& Handler, G. 2000, A\&A, 357, 981

Ryabchikova, T. A., Savanov, I. S., Malanushenko, V. P., \& Kudryavtsev, D. O. 2001, Astron. Rep., 45, 382

Savanov, I. S., Malanushenko, V. P., \& Ryabchikova, T. A. 1999, Astron. Lett., 25, 802

Seaton, M. J., Zeippen, C. J., Tully, J. A., et al. 1992, Rev. Mex. A\&A, 23, 19

Wade, G. A., Ryabchikova, T. A., Bagnulo, S., \& Piskunov, N. 2001, in Magnetic fields across the Hertzprung-Russel diagram, ed. G. Mathys, S. K. Solanki, \& T. Wickramasinghe, Santiago, Chile, in press

Weiss, W. W., Ryabchikova, T. A., Kupka, F., et al. 2000, in Impact of Large-Scale Surveys on Pulsating Star Research, ed. L. Szabados, \& D. W. Kurtz, ASP Conf. Ser., 203, 487 